\title{
COUNTABLE INJECTIVE MODULES ARE SIGMA INJECTIVE
}

\author{
CHARLES MEGIBBEN
}

\begin{abstract}
In this note we show that a countable injective module is $\Sigma$-injective and consequently a ring $R$ is left noetherian if the category of left $R$-modules has a countable injective cogenerator. Our proof can be modified to establish the corresponding result for quasi-injective modules. We also give an example of a nonnoetherian commutative ring $R$ such that the category of $R$-modules has a countable cogenerator.
\end{abstract}

We let $R$ denote an arbitrary ring with identity and $M$ a unital left $R$-module. Recall that $M$ is injective if and only if for each left ideal $I$ of $R$ and each $R$-homomorphism $f: I \rightarrow M$ there is a $y \in M$ such that $f(r)=r y$ for all $r \in I$. If $X$ is a subset of $M$, then $\mathfrak{I}_{R}(X)$ is the left ideal consisting of those $r \in R$ such that $r x=0$ for all $x \in X$. Similarly if $I$ is a subset of $R$, we let $\mathfrak{r}_{M}(I)=\{x \in M$ : $I x=0\}$. If an arbitrary direct sum of copies of $M$ is injective, then $M$ is said to be $\Sigma$-injective. Faith [4] has shown that an injective module $M$ is $\Sigma$-injective if and only if the ascending chain condition holds for the left annihilator ideals $\mathfrak{l}_{R}(X)$.

\section{TheOREM. A countable injective module is $\Sigma$-injective.}

Proof. Let $y_{1}, y_{2}, \ldots, y_{n}, \ldots$ be an enumeration of the elements of the countable injective $R$-module $M$. Assume by way of contradiction that there exists a strictly ascending chain $I_{1} \subset I_{2} \subset \ldots \subset I_{n} \subset \ldots$ of left annihilator ideals. If we let $X_{n}=\mathfrak{r}_{M}\left(I_{n}\right)$, then $I_{n}=\mathfrak{l}_{R}\left(X_{n}\right)$ and in $M$ we have the strictly descending chain $X_{1} \supset X_{2} \supset \cdots \supset X_{n} \ldots$ Moreover if $X=\cap_{n=1}^{\infty} X_{n}$, then $X=r_{M}(I)$ where $I=\cup_{n=1}^{\infty} I_{n}$. We now construct inductively a sequence $b_{1}, b_{2}, \ldots, b_{n}, \ldots$ in $I$ and a corresponding sequence of $R$-homomorphisms $f_{n}: \sum_{i=1}^{n} R b_{i} \rightarrow M$ with $f_{n} \subseteq$ $f_{n+1}$ and $f_{n}\left(b_{n}\right) \neq b_{n} y_{n}$ for all $n$. For $n=1$, we choose a $z_{1} \in X_{1}$ such that $z_{1}-y_{1} \notin X$. Since $X=\mathfrak{r}_{m}(I)$ there is some $b_{1} \in I$ such that $b_{1}\left(z_{1}-y_{1}\right) \neq 0$ and thus the homomorphism $f_{1}: R b_{1} \rightarrow M$ given by right multiplication by $z_{1}$ has the property that $f_{1}\left(b_{1}\right) \neq b_{1} y_{1}$. Now suppose we have found $b_{1}, \ldots, b_{n}$ and $f_{1}, \ldots, f_{n}$ with the desired properties. Since $M$ is injective, there is a $z_{n}$ in $M$ such that $f_{n}(r)=r z_{n}$ for all $r$ in the domain of $f_{n}$. For sufficiently large $m$, we have $b_{1}, \ldots, b_{n}$ in $I_{m}$ and we select $z_{n+1}$ in $X_{m}$ such that $z_{n+1}+z_{n}-y_{n+1} \notin X$. Then there will

Received by the editors July 8, 1980.

1980 Mathematics Subject Classification. Primary 16A52; Secondary 16A33.

Key words and phrases. Injective module, $\Sigma$-injective, cogenerator, noetherian.

(C) 1982 American Mathematical Society 0002-9939/82/0000-0002/\$01.75 
exist some $b_{n+1}$ in $I$ such that $b_{n+1}\left(z_{n+1}+z_{n}-y_{n+1}\right) \neq 0$ and the map $f_{n+1}$ : $\sum_{i=1}^{n+1} R b_{1} \rightarrow M$ given by right multiplication by $z_{n+1}+z_{n}$ has the required properties. Finally to obtain the desired contradiction we note that the supremum $f$ of all the $f_{n}$ 's is a homomorphism from the left ideal $\sum_{i=1}^{\infty} R b_{i}$ into $M$ and therefore there is a $y \in M$ such that $f(r)=r y$ for all $r$ in the domain of $f$. But this yields $b_{n} y=f\left(b_{n}\right)=f_{n}\left(b_{n}\right) \neq b_{n} y_{n}$ for all $n$, contrary to the fact that $y$ must equal some $y_{n}$.

REMARK. The foregoing proof is but a slight modification of the argument given by Lawrence [6] to show that a countable self-injective ring is necessarily quasiFrobenius. As in that paper, this argument can be generalized to show that if $M$ is an injective $r$-module of regular cardinality $m$, then any well-ordered properly ascending chain in $R$ of left annihilators of subsets of $M$ must have length less than $m$.

Recall that $M$ is a cogenerator if each left $R$-module can be imbedded as a submodule of a product of sufficiently many copies of $M$. Since it is easily seen that the left ideal $I$ is the annihilator of a subset of $M$ if (and only if) $R / I$ can be imedded in a product of copies of $M$, every left ideal of $R$ will be the annihilator of a subset of $M$ provided the latter is a cogenerator. Thus we immediately have the following

COROLLARY 1. If the category of left $R$-modules has a countable injective cogenerator, then $R$ is left noetherian.

Let $J$ be the Jacobson radical of $R$. We call $R$ semilocal if $R / J$ is semisimple. For such a ring $R$ we have only finitely many isomorphically distinct simple left $R$-modules $S_{1}, \ldots, S_{n}$ and as an injective cogenerator we have $E\left(S_{1}\right)$ $\oplus \cdots \oplus E\left(S_{n}\right)$ where $E\left(S_{i}\right)$ is the injective envelope of $S_{i}$. Therefore from Corollary 1 we have the following result.

COROllary 2. If $R$ is semilocal and if the injective envelope of each simple left $R$-module is countable, then $R$ is left noetherian.

Since a nilideal in a left noetherian ring is nilpotent and a semiprimary ring is left artinian if and only if it is left noetherian, we can also make the following observation.

COROLlARY 3. If $R$ is a semilocal ring with nil-Jacobson radical and if the injective envelope of each simple left $R$-module is countable, then $R$ is left artinian.

Examples exist showing that "injective cogenerator" cannot be weakened to "cogenerator" in Corollary 1 and "semilocal" is an essential hypothesis in corollary 2. Indeed there exist countable, commutative, nonnoetherian rings $R$ such that for each maximal ideal $P$ of $R$ the localization $R_{p}$ is a rank one discrete valuation ring. For such a ring $R, E(S)$ will be countable for each simple $R$-module $S$ (see [7, Theorem 3.11]) in spite of the fact that $R$ is not noetherian. Moreover as noted in [2] such an $R$ can be constructed in which exactly one maximal ideal fails to be finitely generated. Under these circumstances $R$ can contain only countably many maximal ideals which in turn give rise to countably many isomorphically distinct 
simple $R$-modules $S_{1}, S_{2}, \ldots, S_{n}, \ldots$ Then the countable module $M=E\left(S_{1}\right) \oplus$ $E\left(S_{2}\right) \oplus \cdots \oplus E\left(S_{n}\right) \oplus \ldots$ is a cogenerator (see, for example, $\left.[1,18.16]\right)$, but it is not injective by Corollary 1 since $R$ is not noetherian.

Finally we wish to note that the proof of our theorem can easily be modified to yield the same conclusion for countable quasi-injective modules. Recall that $M$ is quasi-injective if each homomorphism $f: N \rightarrow M$ with $N$ a submodule of $M$ extends to an endomorphism of $M$. It is not difficult to generalize a result of Fuchs [5] in order to show that $M$ is quasi-injective if and only if it satisfies the following condition: If $I$ is a left ideal of $R$ and if $f: I \rightarrow M$ is an $R$-homomorphism with $\operatorname{Ker} f \supset \mathfrak{I}_{R}(F)$ for some finite subset $F$ of $M$, then there is a $y \in M$ such that $f(r)=r y$ for all $r \in I$. Then armed with the characterization of $\Sigma$-quasi-injective modules given in [3], one can readily carry out the desired proof that countable quasi-injective modules are $\Sigma$-quasi-injective.

\section{REFERENCES}

1. F. Anderson and K. Fuller, Rings and categories of modules, Springer-Verlag, Berlin, Heidelberg and New York, 1974.

2. J. Arnold and R. Gilmer, Idempotent ideals and unions of nets of Prüfer domains, J. Sci. Hiroshima Univ. Ser. A-I 31 (1967), 131-145.

3. A. Cailleau and G. Renault, Etude des modules $\Sigma$-quasi-injectifs, C. R. Acad. Sci. Paris A-B 270 (1970), 1391-1394.

4. C. Faith, Rings with ascending condition on annihilators, Nagoya Math. J. 27 (1966), 179-191.

5. L. Fuchs, On quasi-injective modules, Scuola Norm. Sup. Pisa 23 (1968), 541-546.

6. J. Lawrence, A countable self-injective ring is quasi-Frobenius, Proc. Amer. Math. Soc. 65 (1977), 217-220.

7. E. Matlis, Injective modules over noetherian rings, Pacific J. Math. 8 (1958), 511-528.

Department of Mathematics, Vanderbilt University, NashVille, TenNessee 37235 